Pesq. Vet. Bras. 37(9):1025-1031, setembro 2017 DOI: $10.1590 / \mathrm{S} 0100-736 X 2017000900019$

\title{
Relação espacial entre a falange distal e o estojo córneo em éguas Campolinas jovens com e sem sinais de obesidade ${ }^{1}$
}

\begin{abstract}
Jéssica F. Magalhães², Leonardo R. Lima², Cahuê F.R. Paz², Sérgio S. Rocha Junior², Álvaro P.L. Oliveira², Patrícia C. Duarte ${ }^{2}$, Luiz A. do Lago ${ }^{3}$ e Rafael R. Faleiros ${ }^{3 *}$

ABSTRACT.- Magalhães J.F., Lima L.R., Paz C.F.R., Rocha Junior S.S., Oliveira A.P.L., Duarte P.C., Lago L.A. \& Faleiros R.R. 2017. [Spatial relationship between the distal phalanx and the hoof capsule in young Campolina mares with and without obesity.] Relação espacial entre a falange distal e o estojo córneo em éguas Campolinas jovens com e sem sinais de obesidade. Pesquisa Veterinária Brasileira 37(9):1025-1031. Escola de Veterinária, Universidade Federal de Minas Gerais, Av. Presidente Antônio Carlos 6627, Cx. Postal 567, Pampulha, Belo Horizonte, MG 31270-901, Brazil. E-mail: faleirosufmg@gmail.com

Previous studies have demonstrated radiographic changes in the hoof of obese horses. The objective was to study through radiographic evaluation the spatial relationship between the hoof capsule and the distal phalanx of Campolina mares with and without obesity. Twenty-two Campolina mares 3 to 5 years old were used. We analyzed adiposity variables and radiographic measurements of the hoof of both forelimbs of mares with body condition score from 5 to $7 / 9$ (Control group) and from 8 to 9 (Obese group). Comparisons were made between groups using the Student $t$ test for parametric variables and the Mann-Whitney test for the nonparametric ones. Pearson or Spearman tests were used to evaluate the correlation among parametric and nonparametric variables respectively $(\mathrm{P}<0.05)$. Evidences of changes in the spatial relationship between the hoof capsule and the distal phalanx were observed in obese Campolina young mares.
\end{abstract}

INDEX TERMS: Spatial relationship, distal phalanx, hoof capsule, Campolina mares, obesity, hoof, body condition, equine, laminitis.

RESUMO.- Estudos prévios têm demonstrado alterações radiográficas em cascos de equinos obesos. Os objetivos foram estudar, com avaliação radiográfica, a relação espacial entre estojo córneo e falange distal de éguas jovens da raça Campolina com e sem obesidade. Foram utilizadas 22 éguas entre três e cinco anos de idade, sendo analisadas varáveis de adiposidade e medidas radiográficas dos cascos dos membros torácicos de éguas com escore corporal de 5 a $7 / 9$ (Grupo Controle) e de 8 a 9 (Grupo Obeso). Foram feitas comparações entre os grupos e correlacionaram-se as variáveis de adiposidade entre si, variáveis adiposidade com variáveis casco e variáveis de casco entre si. Utilizou-

\footnotetext{
${ }^{1}$ Recebido em 26 de dezembro de 2015

Aceito para publicação em 27 de janeiro de 2017.

${ }^{2}$ Curso de Pós-Graduação, Universidade Federal de Minas Gerais (UFMG), Av. Presidente Antônio Carlos 6627, Cx. Postal 567, Pampulha, Belo Horizonte, MG 31270-901, Brasil.

${ }^{3}$ Escola de Veterinária, UFMG, Av. Presidente Antônio Carlos 6627, Caixa Postal 567, Pampulha, Belo Horizonte, MG 31270-901, Brasil. *Autor para correspondência: faleirosufmg@gmail.com
}

-se o teste $t$ de Student para variáveis paramétricas e o teste Mann-Whitney para as não-paramétricas, para as medidas de correlação, utilizou o teste de Pearson para duas amostras paramétricas e o teste de Spearman para comparações que envolvam pelo menos uma variável não paramétrica $(\mathrm{P}<0,05)$. Os resultados demonstraram que éguas Campolinas obesas, ainda jovens, já apresentam indícios de alteração na relação espacial entre estojo córneo e falange distal.

TERMOS DE INDEXAÇÃO: Relação espacial, falange distal, estojo córneo, éguas Campolinas, obesidade, casco, escore corporal, equino, laminite.

\section{INTRODUÇÃo}

A laminite é a segunda afecção que mais promove mortes em equinos, atrás apenas da síndrome cólica. Dentre as doenças do casco, é a mais grave, causando alterações irreversíveis às estruturas e enorme sofrimento ao animal (Pollitt 2008).

Muitos pesquisadores vêm estudando, há muitos anos, a laminite em equinos. Apesar disso, ainda pouco se sabe, 
principalmente, a respeito da fisiopatologia e tratamento. As teorias sobre a fisiopatologia da laminite de maior destaque, atualmente, são a inflamatória e a endocrinopática (Asplin et al. 2007, Pollitt 2008, Katz \& Bailey 2012).

Dentre as doenças hormonais, a síndrome metabólica é considerada como uma das principais causas de laminite em equinos em todo o mundo. 0 termo síndrome metabólica equina (SME) descreve o aumento da adiposidade associada à disfunção insulínica (DI), que caracteriza as alterações endócrinas (Frank et al. 2010, Paz et al. 2013).

Equinos obesos desenvolvem um quadro com características diferentes da laminite causada por doenças sistêmicas ou sepse. Seu curso é subclínico, ocorrendo alterações importantes nas estruturas do casco, mesmo antes do aparecimento dos primeiros sintomas. Com isso, o diagnóstico precoce é a principal forma de controle desse tipo de laminite (Walsh et al. 2009).

Em estudos prévios do nosso Grupo de Pesquisa, trabalhando com as raças nacionais Crioulo, Mangalarga Marchador e mestiços de Brasileiro de Hipismo, foram encontradas correlações positivas entre o aumento da adiposidade e alterações das medidas do dígito (Paz et al. 2013, Magalhães et al. 2014, Xavier et al. 2014). Entretanto, não se encontram estudos sobre a raça Campolina.

Os objetivos foram estudar, com avaliação radiográfica, a relação espacial entre o estojo córneo e a falange distal de éguas jovens da raça Campolina. Comparar fêmeas jovens com e sem sinais de obesidade e avaliar possíveis correlações entre medidas radiográficas do posicionamento da falange distal e sinais de obesidade.

\section{MATERIAL E MÉTODOS}

Foram avaliados 44 cascos dos membros torácicos de fêmeas da raça Campolina, oriundas de diferentes haras de criadores. Inicialmente elas passaram por um exame de claudicação em movimento e pelo exame da pinça de casco, segundo Stashak (2002), no qual, apenas os animais que não tinham claudicação e sensibilidade dolorosa no casco foram utilizados no experimento. A idade dos animais foi fornecida pelos responsáveis que acompanhavam o animal no momento da avaliação. As éguas tinham de 3 a 5 anos.

Os equinos foram avaliados quanto ao escore de condição corporal (ECC) de acordo com graduação proposta por Henneke et al. (1983), que varia de 1 a 9 (1 égua emaciada e 9 éguas extremamente obeso). Com base nessa classificação foram divididos em dois grupos, um grupo controle (ECC de 5 a 7), grupo obeso (ECC 8 a 9). 0 peso e a altura de cada animal foram registrados com fita de pesagem e hipômetro (WalMur Instrumentos Veterinários).

Para verificação da deposição de gordura na região cervical dorsal, conhecida como crista do pescoço, que é um sinal de doença metabólica, foi utilizado uma escala de 0 a $5(0=$ crista não visível nem palpável, 5 = crista tão grande que se inclina permanentemente para um dos lados), de acordo com o descrito por Carter et al. (2009). Ainda com o mesmo objetivo, medidas da circunferência do pescoço, região cervical, foram tomadas em três alturas diferentes em relação ao comprimento total do pescoço, região cervical, com fita métrica, segundo metodologia descrita por Frank et al. (2006). Essas medidas iniciam-se em pontos dorsais e correspondem a $25 \%, 50 \%$ e $75 \%$ deste comprimento.

Para avaliação radiográfica, os cascos dos membros torácicos foram limpos e posicionado sobre dois blocos de madeira, de igual altura. A projeção foi lateromedial, a uma distância padronizada, usando-se um aparelho de raios X (EcoRay Co., Ltd 1060HF, Korea), dotado de distanciador com iluminação laser, com o objetivo de se visualizar a relação espacial entre falange distal e estojo córneo. As exposições foram realizadas sempre com $80 \mathrm{Kv}$ e 2,5mAs. Para possibilitar a identificação radiográfica da superfície dorsal da parede do casco e do plano da banda coronária, um fio metálico de comprimento conhecido foi aderido à superfície dorsal da pinça do casco, com sua extremidade proximal sempre coincidindo com o plano da banda coronária. 0 fio metálico foi posicionado sempre pela mesma pessoa. Cada casco foi radiografado duas vezes, portanto, quatro imagens foram obtidas de cada animal.

As imagens foram digitalizadas por meio um sistema computadorizado e as mensurações feitas através do programa computacional Metron Hoof-Pro ${ }^{4}$. Sempre antes de realizar as mensurações utilizava-se o comprimento do marcador radiopaco para corrigir o fator de ampliação. As quatro imagens de cada animal foram aferidas e o resultado final de cada membro foi a média das duas imagens de cada um dos membros.

De acordo com o programa Metron Hoof- $\mathrm{Pro}^{4}$ e com o que foi descrito por Thrall (2002) e Pollitt (2008), foram obtidas as medidas para as seguintes variáveis: distância entre os planos horizontais entre a banda coronária e o ápice do processo extensor da falange distal (afundamento) (Fig.1A), ângulo palmar (Fig.1A), profundidade ou espessura de sola (Fig.1B), ângulo da parede dorsal do casco (âng. Casco) (Fig.1C), distâncias entre as superfícies dorsais da falange distal e da parede do casco, proximal (CF Prox.) e distal (CF Distal) (Fig.2A), ângulo do eixo casco-quartela (casco-quartela), ângulo entre as superfícies dorsais da falange distal e da parede do casco (rotação) e breakover (Fig.2B).
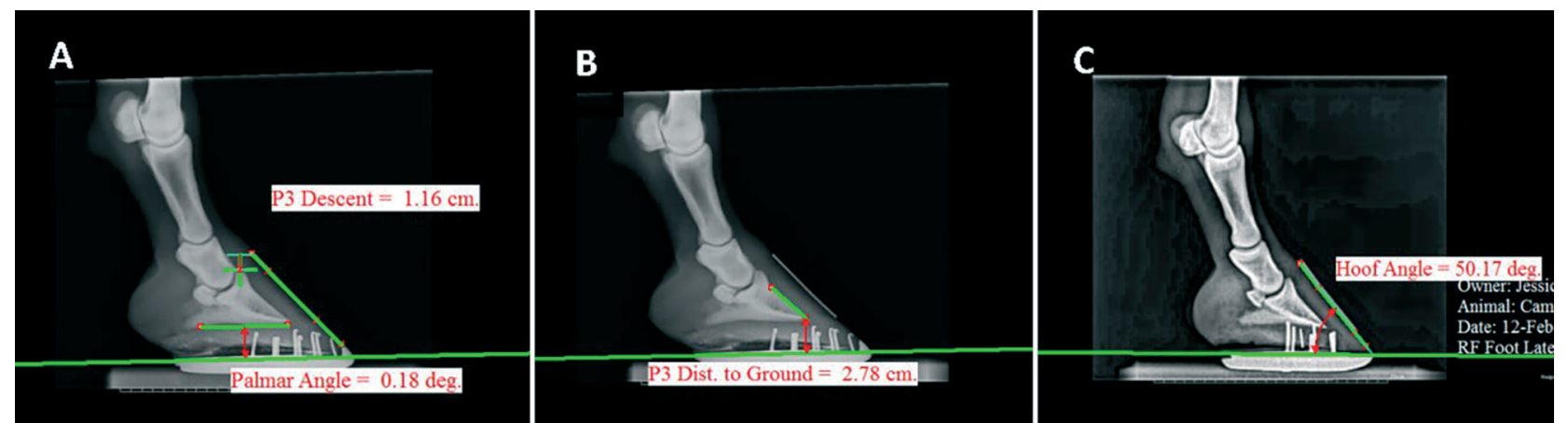

Fig.1. Imagens de mensurações radiográficas realizadas em cascos de membros torácicos de fêmeas da raça Campolina por meio do Software Metron Hoof Pro. (A) Ângulo palmar da falange distal e distância entre os planos horizontais entre a banda coronária e o ápice do processo extensor da falange distal, chamada distância de afundamento. (B) Espessura ou profundidade de sola. (C) Ângulo de casco. 

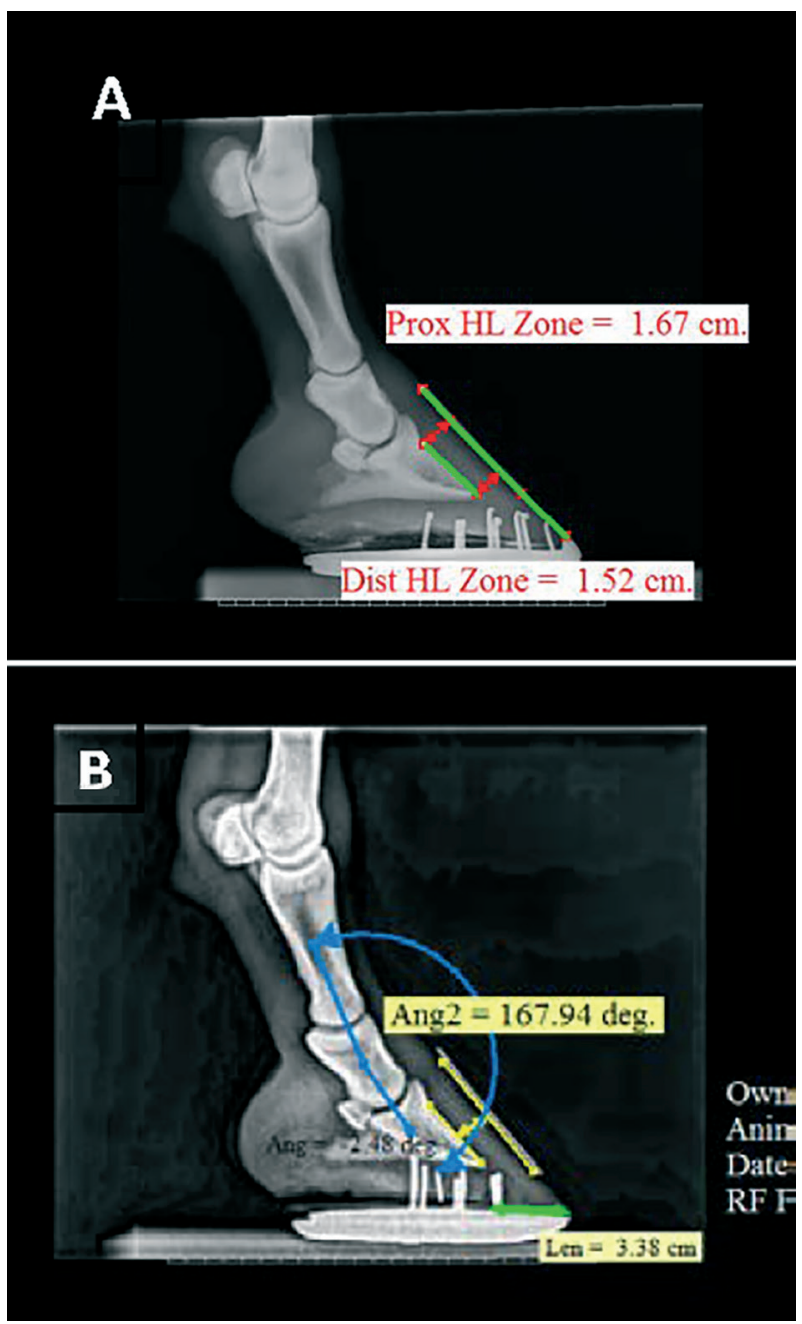

Fig.2. Imagens de mensurações radiográficas realizadas em cascos de membros torácicos de fêmeas da raça Campolina por meio do Software Metron Hoof Pro. (A) Distâncias entre as superfícies dorsais da falange distal e da parede do casco, proximal (CF Prox.) e distal (CF Distal). (B) Ângulo entre as superfícies dorsais da falange distal e da parede do casco (rotação), do ângulo do eixo casco-quartela (casco-quartela) e da distância do breakover.

Para comparação entre os grupos, utilizou-se o teste t de Student para variáveis paramétricas e o teste Mann-Whitney para as não paramétricas. Para as medidas de correlação, utilizou o teste de Pearson para duas amostras paramétricas e o teste de Spearman para comparações que envolvam pelo menos uma variável não paramétrica. Em todos os testes, considerou-se $\mathrm{P}<0,05$. Para efeitos de comparar os graus de correlação, consideraram-se os seguintes níveis de correlação: elevada $(>0,60)$, média (de 0,30 a $0,60)$ e baixa $(<0,30)$.

\section{RESULTADOS E DISCUSSÃO}

Os resultados das comparações entre controles e obesos, para variáveis de adiposidade e mensurações radiográficas do casco estão representados, respectivamente, nos Quadros 1 e 2. Éguas do grupo obeso, tiveram maior peso $(\mathrm{P}=0,04)$, escore $(\mathrm{P}=0,001)$ e espessura de pescoço (região cervical) a $50 \%(\mathrm{P}=0,04)$ do que éguas controles. Tais resultados demonstram aumento de peso e deposição de gordura na região do pescoço em fêmeas jovens da raça campolina.

Semelhante ao verificado no presente estudo, maior deposição de gordura na região do pescoço também foi relatado em fêmeas obesas de raças nacionais como Mangalarga Marchador (Magalhães et al. 2014) e Crioulo (Paz et al. 2013). A média aqui encontrada para o escore de pescoço das éguas jovens obesas $(3,5 \pm 0,52$ graus $)$ foi inclusive superior ao encontrado por Paz et al. (2013) em éguas Crioulas obesas ( 3,3 graus). 0 acúmulo de tecido adiposo ao longo da região cervical dorsal, comumente referida como crista do pescoço em equinos obesos, é preocupante devido à sua associação com alterações metabólicas como hiperinsulinemia e laminite (Johnson 2002, Treiber et al. 2006). Síndrome metabólica equina e doença de Cushing têm sido relatadas em equinos com escore de crista de pescoço acima de 3 (Walsh et al. 2009).

Avaliando os valores das variáveis casco, ainda comparando grupo obeso com grupo controle de éguas jovens (Quadro 2), a única variável com diferença significativa foi espessura de sola $(P=0,03)$. Essa diferença pode ser explicada porque todas as éguas do grupo obeso estavam recentemente casqueadas, enquanto que mais de um terço das éguas do grupo controle era constituído de éguas a pasto, sem casqueamento nos dias que antecederam o exame. Kummer et al. (2006) encontraram uma alteração na espessura de sola de $13 \%$ após casqueamento, que foi exatamente o percentual de alteração encontrado ao comparar o valor das médias das éguas Campolinas jovens do grupo obeso e controle $(19 \pm 4,1 \mathrm{~mm}$ e $16,6 \pm 2,2 \mathrm{~mm})$. Apesar da diferença, ambos valores médios estão dentro do considerado normal por Redden (2003), que considera normal uma espessura de sola maior que $15 \mathrm{~mm}$.

Quadro 1. Valores mínimo, máximo, média e desvio padrão das variáveis adiposidade do grupo controle $(n=16$ cascos) e do grupo obeso $(n=28$ cascos) de éguas Campolina com três a cinco anos de idade

\begin{tabular}{|c|c|c|c|c|c|c|c|c|c|}
\hline \multirow[t]{2}{*}{ Variável } & \multicolumn{4}{|c|}{ Grupo controle $(n=16)$} & \multicolumn{4}{|c|}{ Grupo obeso $(n=28)$} & \multirow{2}{*}{$\begin{array}{l}\text { Valor } \\
\text { de P }\end{array}$} \\
\hline & Mínimo & Máximo & Média & Desvio Padrão & Mínimo & Máximo & Média & Desvio Padrão & \\
\hline Idade (anos) & 3 & 5 & 3,8 & 0,22 & 3,5 & 5 & 4,21 & 0,61 & 0,15 \\
\hline Peso (Kg) & 415 & 510 & 464,3 & 36 & 440 & 580 & 502,5 & 39,78 & 0,04 \\
\hline Altura (m) & 1,45 & 1,59 & 1,52 & 0,05 & 1,44 & 1,65 & 1,55 & 0,05 & 0,29 \\
\hline ECC (grau) & 5 & 7 & 6,38 & 0,74 & 8 & 8 & 8 & 0 & 0,00 \\
\hline Esc. Pescoço (grau) & 2 & 3 & 2,57 & 0,53 & 3 & 4 & 3,5 & 0,52 & 0,00 \\
\hline Comp. Pesc. $(\mathrm{cm})$ & 83 & 98 & 91,13 & 5,67 & 82 & 102 & 92,21 & 6,44 & 0,69 \\
\hline Diam. Pesc. 25\% (cm) & 75 & 80,5 & 77,44 & 2,16 & 54 & 85 & 77,43 & 7,46 & 1,00 \\
\hline Diam. Pesc. 50\% (cm) & 87 & 101 & 93,88 & 5,03 & 91 & 109 & 99,43 & 6,21 & 0,04 \\
\hline Diam. Pesc. 75\% (cm) & 109 & 122 & 114,6 & 5,15 & 109 & 130 & 118,8 & 5,93 & 0,11 \\
\hline
\end{tabular}


Quadro 2. Valores mínimo, máximo, média e desvio padrão das variáveis casco do grupo controle ( $n=16$ cascos) e do grupo obeso $(n=28$ cascos) de éguas de éguas Campolina com três a cinco anos de idade

\begin{tabular}{|c|c|c|c|c|c|c|c|c|c|}
\hline \multirow[t]{2}{*}{ Variável } & \multicolumn{4}{|c|}{ Grupo controle $(n=16)$} & \multicolumn{4}{|c|}{ Grupo obeso (n=28) } & \multirow{2}{*}{$\begin{array}{l}\text { Valor } \\
\text { de P }\end{array}$} \\
\hline & Mínimo & Máximo & Média & Desvio Padrão & Mínimo & Máximo & Média & Desvio Padrão & \\
\hline Ang. Palmar & 0,52 & 6,76 & 3,07 & 1,53 & $-2,47$ & 5,27 & 4,26 & 2,03 & 0,30 \\
\hline Afundamento (mm) & 7,5 & 14,9 & 10,6 & 2 & 6,1 & 14,5 & 11 & 2 & 0,53 \\
\hline Esp. Sola (mm) & 13,4 & 21,1 & 16,6 & 2,2 & 11,1 & 27,2 & 19 & 4,1 & 0,03 \\
\hline Ang. Casco & 46,1 & 52,48 & 49,88 & 1,71 & 44,48 & 54,32 & 49,82 & 2,75 & 0,94 \\
\hline CF Prox. (mm) & 14,5 & 20,3 & 17,2 & 1,8 & 15,9 & 20,9 & 18,2 & 1,4 & 0,06 \\
\hline CF Dist. (mm) & 12,7 & 18,7 & 15,7 & 1,8 & 14,1 & 19,5 & 16,3 & 1,4 & 0,17 \\
\hline Rotação P3 (âng) & $-5,35$ & $-0,65$ & $-2,93$ & 1,35 & $-5,45$ & $-0,08$ & $-2,81$ & 1,29 & 0,77 \\
\hline Breakover $(\mathrm{cm})$ & 2,21 & 3,79 & 2,94 & 0,39 & 2,36 & 3,74 & 3,14 & 0,44 & 0,14 \\
\hline Casco-Quartela (âng) & 154,8 & 183,7 & 167,5 & 9,23 & 150,7 & 176,3 & 163,5 & 5,26 & 0,07 \\
\hline
\end{tabular}

A ausência de casqueamento recente em parte das éguas do grupo controle é uma limitação do presente estudo, que dificulta sua futura reprodução. De fato, algumas medidas da relação espacial entre falange distal e estojo córneo podem ser influenciadas pelo casqueamento recente como ângulo palmar, ângulo de rotação, distância de breakover e espessura de sola. Entretanto, o fato de que das oito varáveis estudadas apenas uma (espessura de sola) foi estatisticamente diferente e de que não houve diferença em variáveis não afetadas pelo casqueamento (como distância de afundamento) demonstram que este viés não interferiu no principal objetivo do estudo, de verificar possível interferência da obesidade na relação espacial entre casco e falange distal.

A distância proximal encontrada entre o casco e a falange das éguas Campolinas jovens foi em torno de $18 \mathrm{~mm}$ e a distal em torno de 16 a $17 \mathrm{~mm}$. Esse resultado corrobora com a constatação de que a distância casco-falange distal pode ser menor do que a proximal, devido ao ângulo mais agudo da falange distal com o solo comparado com

Quadro 3. Correlações entre as variáveis adiposidade, éguas até 5 anos de idade $(n=44$ cascos)

\begin{tabular}{|c|c|c|c|c|c|c|c|c|c|}
\hline & & ECC & $\begin{array}{l}\text { Peso } \\
(\mathrm{Kg})\end{array}$ & $\begin{array}{l}\text { Altura } \\
(\mathrm{m})\end{array}$ & Esc. Pesc. & $\begin{array}{c}\text { Comp. Pesc. } \\
\text { (cm) }\end{array}$ & $\begin{array}{l}25 \% \\
(\mathrm{~cm}) \\
\end{array}$ & $\begin{array}{l}50 \% \\
(\mathrm{~cm})\end{array}$ & $\begin{array}{l}75 \% \\
(\mathrm{~cm}) \\
\end{array}$ \\
\hline Idade & $\mathrm{r}$ & 0,36 & 0,10 & 0,29 & 0,37 & 0,10 & 0,22 & 0,06 & 0,06 \\
\hline & $\mathrm{p}$ & $\begin{array}{l}0,02^{*} \\
*\end{array}$ & 0,49 & 0,05 & $\begin{array}{c}0,02 \\
*\end{array}$ & 0,52 & 0,15 & 0,71 & 0,70 \\
\hline ECC & $\mathrm{r}$ & & 0,40 & 0,26 & 0,66 & 0,00 & 0,17 & 0,38 & 0,35 \\
\hline & $\mathrm{p}$ & & $\begin{array}{c}0,01 \\
* *\end{array}$ & 0,09 & $\begin{array}{c}\mathrm{P}<0,00 \\
* * *\end{array}$ & 0,98 & 0,28 & $\begin{array}{c}0,01 \\
*\end{array}$ & $\begin{array}{l}0,02 \\
*\end{array}$ \\
\hline Peso & $\mathrm{r}$ & & & 0,73 & 0,48 & 0,40 & 0,49 & 0,71 & 0,76 \\
\hline (kg) & $\mathrm{p}$ & & & $\begin{array}{c}\mathrm{P}<0,00 \\
* * *\end{array}$ & $\begin{array}{c}0,00 \\
* *\end{array}$ & $\begin{array}{c}0,01 \\
* *\end{array}$ & $\begin{array}{l}0,00 \\
* * *\end{array}$ & $\begin{array}{c}\mathrm{P}<0,00 \\
* * *\end{array}$ & $\begin{array}{c}\mathrm{P}<0,00 \\
* * *\end{array}$ \\
\hline Altura & $r$ & & & & 0,51 & 0,41 & 0,26 & 0,61 & 0,69 \\
\hline$(\mathrm{m})$ & $\mathrm{p}$ & & & & $\begin{array}{c}0,00 \\
* * *\end{array}$ & $\begin{array}{c}0,01 \\
* *\end{array}$ & 0,09 & $\begin{array}{c}\mathrm{P}<0,00 \\
* * *\end{array}$ & $\begin{array}{c}\mathrm{P}<0,00 \\
* * *\end{array}$ \\
\hline Esc. & $\mathrm{r}$ & & & & & 0,29 & 0,30 & 0,60 & 0,65 \\
\hline Pesc. & $\mathrm{p}$ & & & & & 0,07 & 0,06 & $\begin{array}{c}\mathrm{P}<0,00 \\
* * *\end{array}$ & $\begin{array}{c}\mathrm{P}<0,00 \\
* * *\end{array}$ \\
\hline $\begin{array}{l}\text { Comp. } \\
\text { Pesc. }\end{array}$ & $r$ & & & & & & 0,36 & 0,42 & 0,61 \\
\hline$(\mathrm{cm})$ & $\mathrm{p}$ & & & & & & $0,02 *$ & 0,00 & $\mathrm{P}<0,00$ \\
\hline $\begin{array}{l}25 \% \\
\text { (cm) }\end{array}$ & $r$ & & & & & & & $\begin{array}{l}0,52 \\
0,00 \\
* * *\end{array}$ & $\begin{array}{l}0,52 \\
0,00 \\
* * *\end{array}$ \\
\hline $\begin{array}{l}50 \% \\
(\mathrm{~cm})\end{array}$ & $\begin{array}{l}r \\
p\end{array}$ & & & & & & & & $\begin{array}{c}0,80 \\
\mathrm{P}<0,00 \\
* * *\end{array}$ \\
\hline
\end{tabular}

$\mathrm{r}>0,60=$ correlação alta; $\mathrm{r} 0,30$ a $0,60=$ correlação média; $\mathrm{r}<0,30=$ correlação baixa. * $\mathrm{P}<0,05$; ${ }^{* *} \mathrm{P}<0,01$; ${ }^{* * *} \mathrm{P}<0,001$.
Quadro 4. Correlações entre as variáveis de adiposidade e variáveis casco, éguas até 5 anos de idade ( $n=44$ cascos)

\begin{tabular}{|c|c|c|c|c|c|c|c|c|c|c|}
\hline & & $\begin{array}{l}\text { Ang. } \\
\text { Palm. }\end{array}$ & id & $\begin{array}{l}\text { Esp. } \\
\text { Sola }\end{array}$ & $\begin{array}{c}\text { Ang. } \\
\text { Casco }\end{array}$ & $\begin{array}{c}\mathrm{CF} \\
\text { Prox. }\end{array}$ & $\begin{array}{l}\text { CF } \\
\text { Dist. }\end{array}$ & Rota & $\begin{array}{l}\text { Casco } \\
\text {-quart }\end{array}$ & akov \\
\hline \multirow{2}{*}{ Idade } & $\mathrm{r}$ & $-0,07$ & 0,11 & 0,04 & $-0,29$ & 0,21 & 0,40 & 0,22 & $-0,03$ & 0,27 \\
\hline & $\mathrm{p}$ & 0,66 & 0,46 & 0,78 & 0,06 & 0,16 & $\begin{array}{c}0,01 \\
* *\end{array}$ & 0,16 & 0,86 & 0,07 \\
\hline \multirow[t]{2}{*}{ ECC } & $r$ & $-0,10$ & 0,18 & 0,39 & 0,05 & 0,31 & 0,24 & 0,06 & $-0,27$ & 0,22 \\
\hline & $\mathrm{p}$ & 0,52 & 0,26 & $\begin{array}{c}0,01 \\
* *\end{array}$ & 0,77 & $\begin{array}{c}0,04 \\
*\end{array}$ & 0,12 & 0,70 & 0,08 & 0,15 \\
\hline Peso & $r$ & 0,07 & 0,22 & 0,17 & 0,19 & 0,36 & 0,30 & 0,05 & $-0,06$ & 0,11 \\
\hline (kg) & $\mathrm{p}$ & 0,65 & 0,16 & 0,26 & 0,22 & $\begin{array}{c}0,02 \\
*\end{array}$ & 0,05 & 0,74 & 0,71 & 0,49 \\
\hline Altura & $r$ & $-0,14$ & 0,27 & 0,25 & 0,01 & 0,32 & 0,40 & 0,18 & $-0,13$ & 0,33 \\
\hline$(\mathrm{m})$ & $\mathrm{p}$ & 0,36 & 0,08 & 0,10 & 0,94 & 0,03 & $\begin{array}{c}0,01 \\
* *\end{array}$ & 0,24 & 0,40 & $\begin{array}{c}0,03 \\
*\end{array}$ \\
\hline Esc. & $r$ & $-0,03$ & 0,11 & 0,21 & 0,10 & 0,37 & 0,49 & 0,20 & $-0,15$ & 0,29 \\
\hline Pesc & $\mathrm{p}$ & 0,85 & 0,49 & 0,19 & 0,52 & $\begin{array}{c}0,02 \\
*\end{array}$ & $\begin{array}{l}0,00 \\
* * *\end{array}$ & 0,20 & 0,36 & 0,06 \\
\hline Comp. & $r$ & $-0,15$ & 0,24 & $-0,11$ & $-0,32$ & 0,46 & 0,41 & -0 , & & 0,25 \\
\hline Pesc. & $\mathrm{p}$ & 0,33 & 0,11 & 0,46 & $\begin{array}{c}0,03 \\
*\end{array}$ & $\begin{array}{c}0,00 \\
* *\end{array}$ & $\begin{array}{c}0,01 \\
* *\end{array}$ & 0,41 & 0,93 & 0,10 \\
\hline $25 \%$ & $r$ & 0,23 & 0,21 & 0,10 & 0,24 & 0,11 & $-0,03$ & $-0,26$ & 0,1 & $-0,05$ \\
\hline & $\mathrm{p}$ & 0,14 & 0,16 & 0,50 & 0,11 & 0,49 & 0,82 & 0,09 & 0,27 & 0,73 \\
\hline $50 \%$ & $\mathrm{r}$ & 0,16 & 0,10 & 0,25 & 0,38 & 0,13 & 0,15 & $-0,03$ & 0,09 & $-0,02$ \\
\hline$(\mathrm{cm})$ & $\mathrm{p}$ & 0,29 & 0,53 & 0,11 & $\begin{array}{c}0,01 \\
*\end{array}$ & 0,38 & 0,33 & 0,84 & 0,57 & 0,92 \\
\hline & $r$ & 0,12 & 0,25 & 0,08 & 0,25 & 0,34 & 0,32 & $-0,02$ & $-0,16$ & 0,06 \\
\hline$(\mathrm{cm})$ & $\mathrm{p}$ & 0,43 & 0,10 & 0,62 & 0,11 & 0,02 & 0,03 & 0,89 & 0,31 & 0,68 \\
\hline
\end{tabular}

$\mathrm{r}>0,60=$ correlação alta; $\mathrm{r} 0,30$ a $0,60=$ correlação média; $r<0,30=$ correlação baixa. ${ }^{*} \mathrm{P}<0,05 ;{ }^{* *} \mathrm{P}<0,01 ;{ }^{* * *} \mathrm{P}<0,001$.

o ângulo da parede dorsal do casco com o solo (Sherlock \& Parks 2013). Além disso, esses valores estão próximos aos encontrados por Magalhães et al. (2014) e Xavier et al. (2014) que foram em média $19,65 \mathrm{~mm}$ e $17 \mathrm{~mm}$, respectivamente. Outros valores foram encontrados na literatura internacional: $<19 \mathrm{~mm}$ para raças menores $(<1,50 \mathrm{~m}$ de altura) (Cripps \& Eustace 1999, Redden 2003, Fraley 2007), 18 a $20 \mathrm{~mm}$ para cavalos de sela "Warmblood" (Sloet Van Oldruitenborgh-Oosterbaan 1999) e média de $20 \mathrm{~mm}$ para trotadores "Standardbreds" (Redden 2003).

Os resultados das correlações das variáveis de adiposidade entre si, as variáveis adiposidade com as variáveis casco e as variáveis casco entre si, de acordo com os Quadros 3 a 5 a seguir.

0 peso dessas éguas teve correlação positiva com todas as variáveis usadas para avaliar a adiposidade. Dessa forma, podemos ter certeza que todas as variáveis foram 
Quadro 5. Correlações entre as variáveis casco, éguas até 5 anos de idade ( $n=44$ cascos)

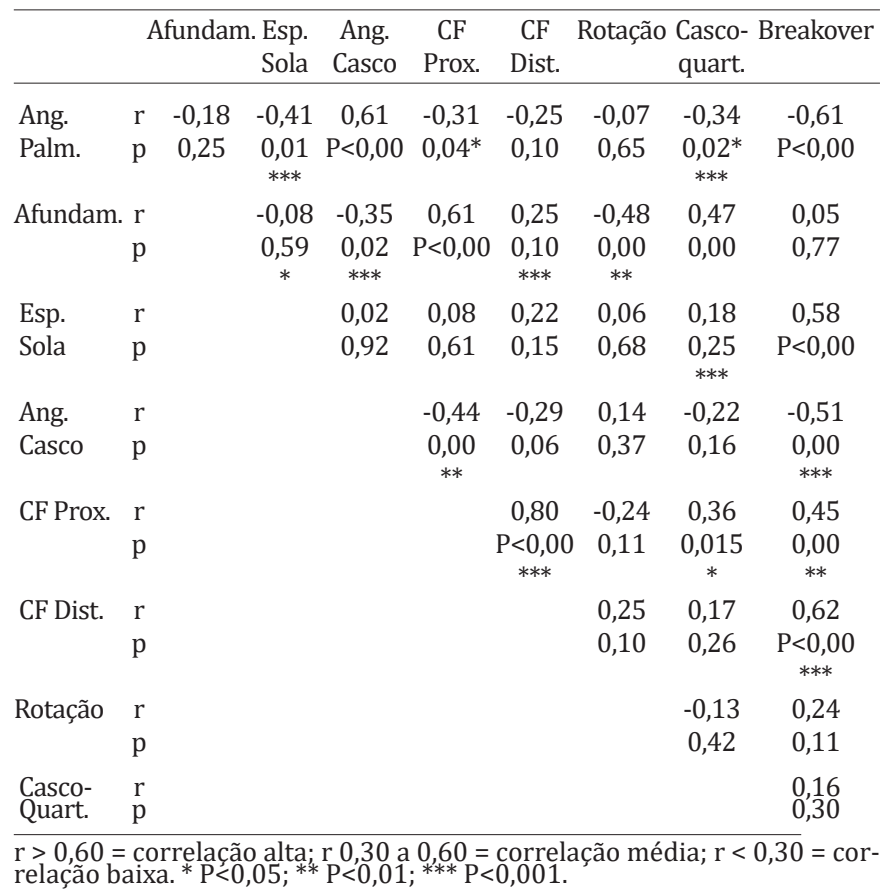

aferidas corretamente, mostrando que quanto maior o peso das éguas, maior acúmulo de gordura elas têm. Além disso, essas correlações positivas médias a altas entre esses parâmetros indicam segurança na utilização desses para determinar adiposidade em éguas Campolinas. Ainda no Quadro 3, pôde-se observar que quanto mais altas eram as éguas maior eram o comprimento e o escore de pescoço e a espessura do pescoço a 50 e 75\% (região cervical).

É esperado que um animal mais alto tenha as estruturas do corpo proprocionalmente maiores. Essa proporção também pode ser vista na correlação encontrada entre o comprimento da região cervical (pescoço) e sua espessura a 25,50 e $75 \%$. A espessura do pescoço (região cervical) a $25 \%$ de éguas campolinas jovens parece ser a região que menos acompanha, em proporção, o comprimento do pescoço e a altura do animal. Mas, ao correlacinar as espessuras do pesoço entre si, encontrou-se alta correlação. Ou seja, o acúmulo de gordura no pescoço (região cervical) dessas éguas jovens é refletido em todo o comprimento do pescoço.

A variável idade correlacionou-se positivamente com as variáveis ECC e escore de pescoço. Uma vez que essas éguas jovens têm de 3 a 5 anos de idade, é natural que ainda estejam em fase de desenvolvimento e acumulando gordura com o avançar da idade. Já o ECC, apresentou uma correlação elevada $(r=0,66)$ com o escore de pescoço (região cervical), média com o peso $(\mathrm{r}=0,40)$ e com o diâmetro do pescoço a $50(\mathrm{r}=0,38)$ e $75 \%(\mathrm{r}=0,35)$. Isso mostra que o ECC pode ser usado com segurança como uma forma de avaliar a adiposidade e que existe uma grande tendência ao acúmulo de adiposidade no pescoço dos equinos, nesses animais, principalmente a 50 e $75 \%$.

Em éguas Mangalarga Marchador, o diâmetro do pescoço a 25 e $50 \%$ foram os que mais apresentaram correlação com os outros parâmetros de adiposidade e o diâmetro a $75 \%$ apresentou baixa correlação com os outros parâmetros (Magalhães et al. 2014). Diferentemente do encontrado nas éguas MM, nas éguas Campolinas o diâmetro a $25 \%$ foi o que apresentou menor correlação com os demais parâmetros de adiposidade e os diâmetros a 50 e 75\%, de um modo geral, apresentaram alta correlação com os outros parâmetros. Essa diferença pode ser atribuída às diferenças morfológicas entre as raças. Ou seja, aparentemente éguas MM tendem a apresentar maior acúmulo de gordura na parte médio-cranial do pescoço e Campolinas na porção médio-caudal do pesoço.

Outros trabalhos demonstraram correlações entre ECC e gordura subcutânea (Gentry et al. 2004, Rodrigues et al. 2011), entre ECC e sensibilidade a insulina (Vick et al. 2007) e entre ECC e concentração de leptina (Buff et al. 2002). A leptina é um indicador endócrino importante da massa adiposa e do estado nutricional, bem como é um relevante regulador de vários aspectos da ingestão de alimentos, crescimento, metabolismo e reprodução (Buff et al. 2002).

No Quadro 4 estão as correlações entre as variáveis adiposidade e as variáveis casco. As varíaveis de casco com maior número de correlações significativas com varíaveis corpóreas foram as distâncias distal e proximal entre superfícies dorsais do casco e falange (CF).

A distância proximal correlacionou-se positivamente de com altura $(r=0,32)$ e comprimento de pescoço $(r=0,46)$, mas também com indicadores de adiposidade como peso $(r=0,36)$, escore corporal $(r=0,31)$, escore de pescoço $(\mathrm{r}=0,37)$ e diâmetro de pescoço a $75 \%(\mathrm{r}=0,34)$. Quanto à distância distal, a situação foi similar com correlações significantes para idade $(r=0,40)$, altura $(r=0,40)$, comprimento de pescoço $(r=0,41)$, mas também para escore de pescoço $(0,49)$ e diâmetro de pescoço $75 \%(r=0,32)$.

Nestas éguas jovens, as correlações com fatores corporais como idade, altura e comprimento de pescoço são indicadoras da influência do desenvolvimento corporal nas distâncias entre casco e falange. Contudo, as várias correlações que houveram com fatores de adiposidade também indicam que a deposição de gordura localizada é proporcional a um aumento da distância em falange distal e parede do casco.

Já está bem documentado em trabalhos internacionais o aumento do risco de desenvolvimento de laminite em cavalos com SME, a qual tem a obesidade como componente primário. Esses estudos mostraram que a obesidade pode estar associada à disfunsão insulínica, além da presença do aspecto inflamatório, causando aumento na concetração circulatória de leptina capaz de estimular a produção de citocinas pró-inflamatórias. Essas citocinas podem alterar a expressão e a ativação de enzimas que regulam a remodelação do tecido, as metaloproteinases, podendo alterar o crescimento do casco. Além disso, existe o envolvimento de alterações vasomotoras como consequência da RI, que podem gerar lesão e separação das lâminas dermais e epidermais do casco, culminando em afundamento e ou rotação da falange distal (Gentry et al. 2004, Asplin et al. 2007, Vick et al. 2007, Frank et al. 2010). Assim as correlações signif- 
icativas entre adiposidade e a distância entre o casco e a falange distal no presente estudo nos permitem supor que alguma possível influência da obesidade no posicionamento da falange distal em relação ao estojo córneo em fêmas jovens da raça Campolina.

No Quadro 5 estão os resultados das correlações entre as variáveis casco. Pôde-se observar que à medida que o ângulo palmar aumenta, um reflexo de talões altos, há aumento concomitante no ângulo do casco $(r=0,61)$ e quebra dorsal do eixo casco-quartela, que diminui seu ângulo $(r=-0,34)$. Tais alterações normalmente promovem maior apoio na região da pinça, que se desgasta mais que os talões, resultando em menos espessura de sola $(r=-0,41)$, menor distância de breakover $(r=-0,62)$.

Outra observação interessante em relação ao Quadro 5, é que a distância de afundamento tem elevada correlação com a distância proximal entre casco e falange $(r=0,61)$. Isto parece começar a refletir uma fragilidade ou mesmo uma proliferação celular na interface derme-epiderme na região próximo-dorsal do casco (Karikoski et al. 2015).

Assim como encontrado no trabalho de Paz et al. (2013) com éguas Crioulas e Magalhães et al. (2014) com éguas $\mathrm{MM}$, esses resultados encontrados no presente trabalho indicam que éguas Campolinas com maior adiposidade também estão mais sujeitas a desenvolver laminite endocrinopática. Apesar de não ter sido realizado exame de dosagem da concentração plasmática de insulina nesse trabalho, para diagnóstico de resistência à insulina, muitos trabalhos já demonstraram a relação entre obesidade e RI em cavalos. Sabe-se, também, que a captação de glicose pelas lâminas do casco não é dependente de insulina (Asplin et al. 2007, Walsh et al. 2009), portanto a lesão não é causada pela falta de glicose e sim, pelas ações vasorreguladoras da insulina (Asplin et al. 2007, Frank et al. 2010). Estudos em humanos têm demonstrado que pessoas que se tornam obesas durante a infância e adolescência tem maior chance de apresentar vários problemas médicos após atingir a fase adulta (Maffeis \& Tatò 2001). Com isso, é esperado que em um próximo estudo com éguas Campolina adultas, haja mais resultados corroborando uma possível associação entre obesidade e laminite.

\section{CONCLUSÕES}

Não se verificaram diferenças de importância clínica entre a relação espacial de falange e estojo córneo entre os grupos controle e obeso de éguas Campolina jovens.

Foi possível verificar correlações positivas entre o acúmulo de gordura localizada na região cervical e a distância dorsal entre estojo córneo e falange.

Novos estudos a serem realizados com éguas Campolinas adultas deverão esclarecer se possíveis diferenças significativas se manifestam com o avançar da idade.

\section{REFERÊNCIAS}

Asplin K.E., Sillence M.N., Pollitt C.C. \& Mcgowan C.M. 2007. Induction of laminitis by prolonged hyperinsulinaemia in clinically normal ponies. Vet. J. 174:530-535.

Buff P., Dodds A., Morrison C., Whitley N.C., McFadin E.L., Daniel J.A., Djiane J. \& Keisler D.H. 2002. Leptin in horses: tissue localization and relation- ship between peripheral concentrations of leptin and body condition. J. Anim. Sci. 80:2942-2948.

Carter R.A., Geor R.J., Burton Staniar W., Cubitt T.A. \& Harris P.A. 2009. Apparent adiposity assessed by standardised scoring systems and morphometric measurements in horses and ponies. Vet. J. 179:204-210.

Cripps P. \& Eustace R. 1999. Radiological measurements from the feet of normal horses with relevance to laminitis. Equine Vet. J. 31:427-432.

Fraley B.T. 2007. Acute Sinker syndrome: diagnosis and therapeutic options. North American Veterinary Conference, p.117-118. Disponível em <http://www.ivis.org/proceedings/navc/2007/LA/044.asp?LA=1> Acesso em 19 mar. 2014.

Frank N., Elliott S.B., Brandt L.E. \& Keisler D.H. 2006. Physical characteristics, blood hormone concentrations, and plasma lipid concentrations in obese horses with insulin resistance. J. Am. Vet .Med. Assoc. 228:13831390.

Frank N., Geor R.J., Bailey S.R., Durham A.E. \& Johnson P.J. 2010. Equine metabolic syndrome. J. Vet. Intern. Med. 24:467-475.

Gentry L., Thompson D. \& Gentry G. 2004. The relationship between body condition score and ultrasonic fat measurements in mares of high versus low body condition. J. Equine Vet. Sci. 24:198-203.

Henneke D.R., Potter G.D., Kreider J.L. \& Yeates B.F. 1983. Relationship between condition score, physical measurements and body fat percentage in mares. Equine Vet. J. 15:371-372.

Johnson P.J. 2002. The equine metabolic syndrome peripheral Cushing's syndrome. Vet. Clin. North Am., Equine Pract. 18(2):271-293. Disponível em <http://www.ncbi.nlm.nih.gov/pubmed/15635908> Acesso em 24 ago. 2015 .

Karikoski N.P., McGowan C.M., Singer E.R., Asplin K.E., Tulamo R.M. \& Patterson-Kane J.C. 2015. Pathology of Natural Cases of Equine Endocrinopathic Laminitis Associated With Hyperinsulinemia. Vet. Pathol. 52(5):945-956.

Katz L.M. \& Bailey S.R. 2012. A review of recent advances and current hypotheses on the pathogenesis of acute laminitis. Equine Vet. J. 44:752761.

Kummer M., Geyer H., Imboden I., Auer J.A. \& Lischer C. 2006. The effect of foot trimming on radiographic measurements of the front feet of normal Warmblood horses. Vet. J. 172:58-66.

Magalhães J.F., Lima I.R., Lima L.R., Varela J.R.C., Vieira R.A.M., Alves G.E.S. \& Faleiros R.R. 2014. Estudo da correlação de medidas radiográficas indicadoras de laminite em éguas da raça Mangalarga Marchador com e sem sinais de sobrepeso. Arq. Bras. Med. Vet. Zootec. 66(4):10231032.

Maffeis C. \& Tatò L. 2001. Long-term effects of childhood obesity on morbidity and mortality. Horm. Res. 55(1):42-45.

Paz C.F.R., Paganela J., dos Santos C.A., Nogueira C.E.W. \& Faleiros R.R. 2013. Relação entre obesidade, insulina plasmática e posicionamento da falange distal em equinos da raça crioula. Arq. Bras. Med. Vet. Zootec. 65:1699-1705.

Pollitt C.C. 2008. Equine Laminitis Current Concepts. Publ. 08/062, Project no. RIRDC UQ-118A. Queensland, Australia.

Redden R.F. 2003. Clinical and radiographic examination of the equine foot. Proc. Am. Assoc. Equine Pract. 49:169-185.

Rodrigues P.G., Raymundo C.M., Souza J.C., Miranda M.C.M.G. \& Rezende A.S.C. 2011. Gordura corporal e eficiência reprodutica em éguas doadoras de embrião Mangalarga Marchador. Ciênc. Agrotec. 35:1002-1008.

Sherlock C. \& Parks A. 2013. Radiographic and radiological assessment of laminitis. Equine Vet. Educ. 25(10):524-535.

Sloet Van Oldruitenborgh-Oosterbaan N.M. 1999. Laminitis in the horse: a review. Vet. Quart. 21:121-127.

Stashak T.S., Hill C., Klimesh R. \& Ovnick G. 2002. Trimming and shoeing for balance and soundness, p.1081-1142. In: Stashak T.S. (Ed.), Adams' Lameness in Horses. 5th ed. Lippincott Williams and Wilkins, Philadelphia.

Thrall D.E. 2002. Textbook of Veterinary Diagnostic Radiology. 4th ed. W.B. Saunders, Philadelphia. 
Treiber K., Kronfeld D., Hess T., Byrd B.M., Splan R.K. \& Staniar W.B. 2006. Evaluation of genetic and metabolic predispositions and nutritional risk factors for pasture-associated laminitis in ponies. J. Am. Vet. Med. Assoc. 228:1538-1545.

Vick M., Adams A., Murphy B., Sessions D.R., Horohov D.W., Cook R.F., Shelton B.J. \& Fitzgerald B.P. 2007. Relationship among inflammatory cytokines, obesity, and insulin sensitivity in the horse. J. Anim. Sci. 85:1144-1155.

Walsh D.M., Mcgowan C.M., Mcgowan T. Lamb S.V., Schanbacher B.J. \&
Place N.J. 2009. Correlation of plasma insulin concentration with laminitis score in a field study of Equine Cushing's Disease and Equine Metabolic Syndrome. J. Equine Vet. Sci. 29:87-94.

Xavier V.F., Lima I.R., Marval C.A.D., Silveira D., Vieira R.A.M., Castro T.D.C., Moura D.A.D., Vilela S.B. \& Faleiros R.R. 2014. Estudo radiográfico do posicionamento da falange distal e suas possíveis correlações com obesidade em equinos de patrulhamento da Polícia Militar do Estado de Minas Gerais. Arq. Bras. Med. Vet. Zootec. 66(3):672-680. 